\title{
The Stern Review and the IPCC fourth assessment report: implications for interaction between policymakers and climate experts. An editorial essay
}

\author{
Klaus Hasselmann • Terry Barker
}

Received: 10 January 2008 / Accepted: 16 April 2008 / Published online: 25 June 2008

(C) The Author(s) 2008

\section{Introduction}

Within just 1 year, the combined impact of the Stern Review (Stern 2007), the Fourth Assessment Report of the UN Intergovernmental Panel on Climate Change (IPCC 2007a, b, c), Al Gore's Oscar-awarded documentary climate film (Gore 2006), and the activities of innumerable nongovernment organizations (NGOs), business councils and citizen groups, have brought about a dramatic increase in the public and political awareness of the climate change problem. Today, the reality and widespread implications of anthropogenic climate change are no longer seriously disputed. Climate change has moved to the top of the political agenda. The question is no longer "Are we experiencing a human-made change in climate?" but "What must we do about it?"

The credit for this sea change must go to a large part to the patient efforts of scientists over many decades, transmitted and amplified by IPCC and gifted communicators. It is therefore most appropriate that the 2007 Nobel Peace Prize has been awarded jointly to the IPCC, chaired by Rajendra Pachauri, and Al Gore.

However, having contributed to the political acceptance of the urgency of the climate problem, climate experts (in the broad sense, including climatologists, economists, social-scientists, legal experts, etc.) now need to accept a major new

K. Hasselmann $(\bowtie)$

Max-Planck-Institute for Meteorology,

Bundestrasse 53, 2000, Hamburg, Germany

e-mail: klaus.hasselmann@zmaw.de

K. Hasselmann

European Climate Forum,

Potsdam, Germany

T. Barker

Cambridge Centre for Climate Change Mitigation Research,

Department of Land Economy,

University of Cambridge, Cambridge, UK 
responsibility. Policymakers face a task of unprecedented complexity and difficulty: the transformation of our present unsustainable global socio-economic system into a sustainable system that will maintain the planet Earth sufficiently close to its present state to ensure the welfare and security of future generations. Moreover, this transformation must be brought well underway with one or two decades.

There exist already many divergent proposals for an international climate agreement to succeed the first assessment period of the Kyoto protocol in 2012. These are being discussed in many national and international conferences, such as the Conference of the Parties of the UN First Framework Convention on Climate Change (UNFCCC) in Bali, December 2007. However, in order to converge towards a common post-Kyoto climate agreement, policymakers will need advice based on objective, rational analyses provided by independent climate experts. And if effective, efficient and equitable policies are to be agreed upon within the limited time window available, the interactions between policymakers and climate experts will need to be much more intimate and on a continuous basis than in the past.

Two problems must be resolved before an effective interaction between climate experts and policymakers can be established: (1) the means of communication and (2) the generation of the relevant information. Let us discuss first the problem of communication.

Climate experts have been highly effective in communicating the scientific basis of climate change to the general public and policymakers through the reports of the first IPCC Working Group (IPCC 2001a, 2007a). However, the influence of IPCC on portfolios of policies and measures (as opposed to government targets and rhetoric) through the second and third IPCC Working Groups on impacts, mitigation potentials and costs (IPCC 2001b, c, 2007b, c) has been much smaller. This is mainly because of the chosen IPCC stance of not engaging in policy debate. IPCC is rightly seen as a politically detached panel, providing "policy-relevant, not policy-prescriptive" information. Its task is to collect and summarize the diverse investigations carried out independently by the scientific community, not to advise on specific questions posed by policymakers.

The effect of the political detachment of IPCC is very evident in the Stern Review. In contrast to the IPCC Reports, the Review is arguably the most comprehensive and powerful document to date on the portfolios of policies required to address the climate change problem. The summary of the scientific evidence of climate change in the first part of the report draws heavily on IPCC Working Group 1 (including a preview of the Fourth Assessment Report, presented later in February, 2007) and is uncontroversial. However, the subsequent sections on impacts and policy had little guidance from the diverse summaries of Working Groups 2 and 3 in the IPCC Third Assessment Report, which were rather dated, or from the texts of the Fourth Assessment Report, which had not been completed. In order to arrive at strong policy recommendations, Stern was therefore forced to freelance by commissioning research and reviews. This has naturally opened the report to criticism. The scientific community should respond constructively to such criticisms by providing stronger scientific foundations for the assessment of climate policy proposals, drawing on the now published full IPCC Reports. ${ }^{1}$

\footnotetext{
${ }^{1}$ The IPCC Synthesis Report was agreed and the Working Group 2 and 3 Reports were published by November 2007.

悬 Springer
} 


\section{An independent climate policy panel}

Although the latest report of Working Group 3 in the Fourth IPCC Assessment Report (IPCC 2007c) focuses more strongly on climate policies, what is needed is a more continuous, ongoing interaction between climate experts and climate policymakers. This could best be established by the creation of an independent UN "Climate Policy Panel" (CPP). Presently, governments are advised by special interest groups or by national bodies, such as the UK Government's Climate Change Committee $^{2}$ or the German Government's Scientific Advisory Committee on the Global Environment. The CCP would need to have a similar remit as such national bodies, but would be an international expert panel assessing policies from a supranational global perspective. It could report directly to the Parties of the UNFCCC, either as a re-constituted or an additional Subsidiary Body. In contrast to the IPCC working groups that report on the current status of climate change issues only every 5 to 6 years, the analyses of the CPP would be documented on a quasi-continuous basis in direct support of the ongoing negotiations of the UNFCCC parties. In addition, the task of CPP would be to analyze other policy proposals, brought into the debate by climate-concerned business networks or NGOs. This would enrich the discussion through further inputs screened by an independent agenda-free scientific panel.

As a panel of experts, the function of CPP would differ from that of the two existing UNFCCC Subsidiary Bodies for Scientific and Technological Advice and for Implementation. These bodies focus on the exchange of information and experience between active policymakers and stakeholders, rather than on the interaction between decision-makers and independent experts engaged in analyzing the impact of proposed climate policies.

The impartiality of the Climate Policy Panel would need to be ensured by agreement of all UNFCCC parties. The panel itself would naturally not be able to resolve the inherent conflicts of interest of climate policy. But by producing quantitative analyses accepted by all parties, it could act as an impartial broker promoting an atmosphere of mutual trust and understanding. This could be an important contribution to overcoming the different perceptions and interests that have often blocked progress in international climate policy. The International Energy Agency (IEA) partly fulfills this role, but there are concerns that it may be perceived as representing the interests of the energy consumers, rather than those of the energy producers, and of society as a whole. Nevertheless, the secretariats of both the IEA and Organization of the Petroleum Exporting Countries maintain global dynamic models that are routinely used to address global climate and energy policies, and which could provide useful input to the analyses of the Climate Policy Panel.

\section{International integrated assessment modelling}

This brings us to the second question: are these and other available models the right tools to provide the information required by policymakers? We argue below that, through their detachment from the political process, the typical integrated

\footnotetext{
${ }^{2}$ See http://www.defra.gov.uk/environment/climatechange/uk/legislation/committee/index.htm.
} 
assessment (IA) models developed so far by the scientific community lack many of the ingredients essential for realistic assessments of climate policy. Most models are based on obsolete concepts of economic equilibrium, full global employment and exogenous technological change. In addition, and more serious when there are competing explanations of system change, many of the detailed models are based on only 1 year's data, which is patently inadequate ${ }^{3}$ for understanding the transformation of the global energy system required to achieve the more stringent targets being proposed by governments-quite apart from the wider implications for the world economy of substantial mitigation action. Before creating a formal UNFCCC Climate Policy Panel, it may therefore be advisable to form first an informal network of integrated assessment modellers that would be willing to jointly develop criteria and methods of evaluation for a common suite of complex, dynamic models designed specifically for climate policy analysis.

Climate policy cannot be considered in isolation. It is inextricably intertwined with broader global concerns, such as north-south inequality, immigration pressure, interregional conflict, terrorism and national security. A meaningful suite of IA models would need to address these more general issues, in addition to traditional economic problems, such as gross domestic product (GDP) growth, technological development, investments in renewable energy, know-how transfer, international trade and competitiveness - as well as other important economic factors such as unemployment, consumer preferences or life-style issues, which are normally ignored in IA models. They should also include a diverse set of instruments for fiscal and monetary policy and incorporate features for testing robustness under uncertainty. Finally, they would need to be sufficiently flexible to enable simulations for a wide range of policy portfolios.

The limited quality and availability of data preclude the construction of a fully specified world model including all of these considerations. Ideally, a comprehensive model should provide assessments of policy impacts at a detailed country level, and offer short-term (quarterly), annual, or 5-yearly projections into the distant future. However, one can create a suite of model sub-systems, each sub-system focusing on a particular aspect of the complete model suite. An individual model sub-system would need to be designed as a model tree, rooted in a simple conceptual model based on a small set of dominant processes, and branching into more complex model versions incorporating further processes. Tree structures clarify whether the ordering of dominant and secondary processes implicit in every model concept is internally consistent. In the process, they define the level beyond which further complexity is no longer justified by the data and the uncertainty of the model parameters. Thus, paradoxically, the development of a full suite of model sub-systems-although still a major challenge-would be considerably simplified by the limited predictability of the climate and socio-economic systems, since this restricts the attainable complexity of the model trees.

To interpret the output of the model simulations, the model suite would need to be augmented by a multi-criteria post-analysis package (Ackerman and Heinzerling 2004) rather than a traditional cost-benefit analysis. This avoids the requirement to translate human life expectancy and ecosystem survival into monetary values, and

\footnotetext{
${ }^{3}$ See (Barker 1998; DeCanio 2003; Beinhocker 2006).

空 Springer
} 
allows for an explicit assessment of risk. ${ }^{4}$ The model suite and post-analysis package would need to be designed as an internally consistent homogeneous system (the difficulties of interpreting the results of an inhomogeneous model ensemble are well documented by model intercomparison studies, cf. Weyant 2000; Löschel 2002; Edenhofer et al. 2006).

In addition to these general considerations, a model suite designed for policy analysis would need to incorporate three key elements. The first is a multi-actor design. This applies both for the simulation of the negotiations between nations and the evolution of the global socio-economic system itself. Different countries make different contributions to climate change, are affected differently by climate change, and have different economic development levels, energy systems and opportunities for low-cost abatement. They also have divergent views on equity. The analysis of climate policy therefore needs to be carried out from the perspectives of many different national or regional negotiators. Similarly, the economic evolution of individual regions within the global socio-economic system is governed by the conflicting goals and actions of many different economic actors, such as business, governments, consumers, media, interest groups, and NGOs.

The second key element is the dynamic nature of the socio-economic system. This is a natural corollary of the multi-actor structure: interactions between actors typically represent dynamic processes. Models starting from the traditional paradigm of economic equilibrium, in which the multi-actor structure is absorbed in a static "invisible hand" with full information and perfect foresight, are inadequate for most climate-policy applications, in which the dominant concerns are non-equilibrium global adjustment processes, such as the transfer of technology, the asymmetrical diffusion of knowledge and information, unemployed resources, and pressures of population migration, especially from rural to urban areas.

The third key element, finally, is the inherent uncertainty and unpredictability of the coupled climate-socio-economic system. This property must be reflected both in the uncertainties of the basic model parameters and in the stochastic evolution of the system trajectories resulting from amplifying feedbacks and unpredictable noise in key system components.

\section{Examples of proposed integrated assessment modelling}

The interplay between these features is best illustrated by some simple examples.

Traditional economic assessments of climate policies have been based on costbenefit analyses (Nordhaus 1994). A climate policy is regarded as beneficial if the discounted sum $S=M+D$ of the mitigation costs $M$ and the residual climate damages $D$ is less than the business-as-usual climate damages $D_{0}$. Thus climate policies below the indifference line $M+D=D_{0}$ in the cost-benefit plane (Fig. 1a) are regarded as acceptable, policies above the line as unacceptable. There are many problems with the cost-benefit approach (van den Bergh 2004), most notably the treatment of climate catastrophe (Weitzman 2008). But apart from these general limitations, a global cost-benefit analysis has little meaning in the context of climate

\footnotetext{
${ }^{4}$ See the Springboard Editorial on the economics of a risk assessment.
} 
a

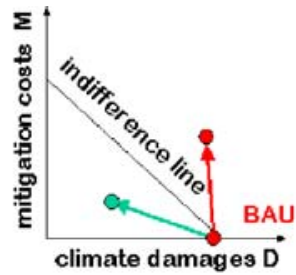

acceptable climate policy: $\longrightarrow 0$

unacceptable climate policy: $\longrightarrow$

b
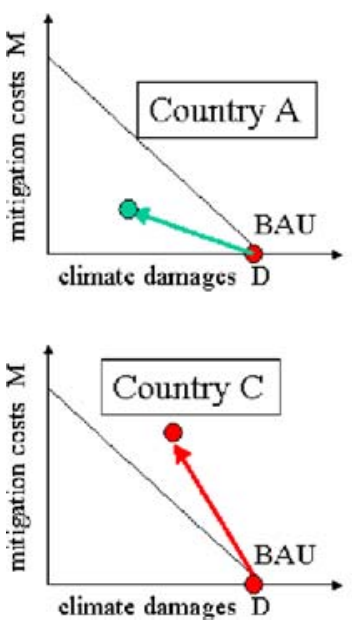
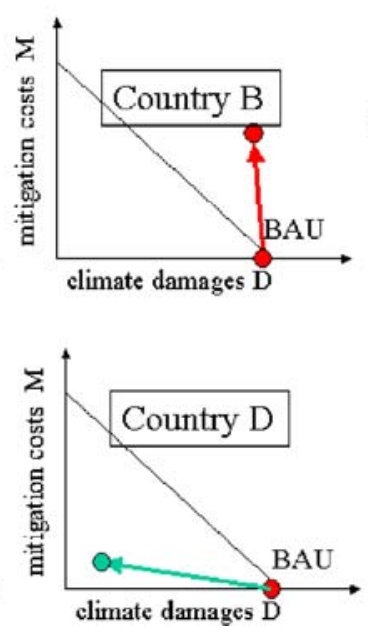

C

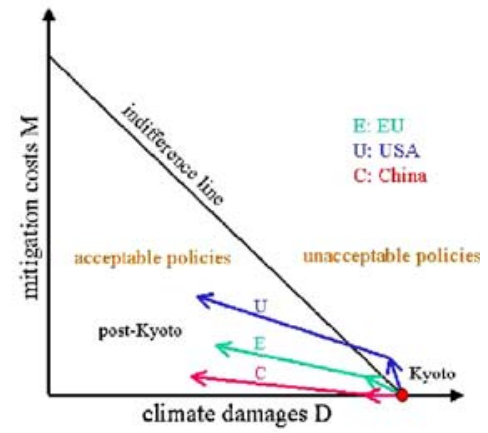

d

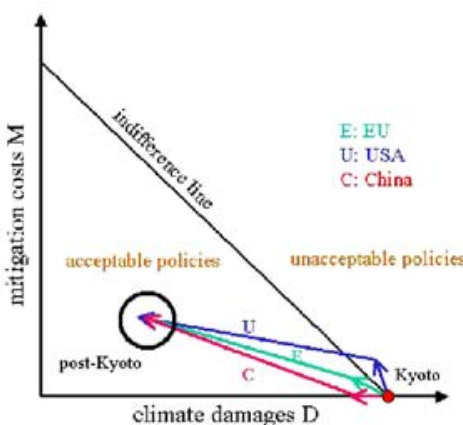

Fig. 1 The climate-policy cost-benefit plane: mitigation costs $M$ versus climate damage costs $D$. a The triangular region below the indifference line $M+D=D_{0}$ (BAU climate damages) represents the space of acceptable (beneficial) climate policies, the region above the line the space of unacceptable (detrimental) climate policies. b The climate-policy assessments of different countries will often lie on different sides of the indifference line. $\mathbf{c}$ The challenge of a post-Kyoto climate agreement will be to bring all arrows for all parties well into the acceptable triangle. $\mathbf{d}$ To be accepted as equitable by all parties, all arrows must furthermore have the same endpoint

negotiations. Each country obtains a different result, dependent on the individual conditions within the country (Fig. 1b).

Thus in the case of the Kyoto protocol, for example, the EU (as all other industrial countries apart from the US and Australia) viewed the 5\% reduction commitment as yielding a net benefit, while the Bush administration concluded that it was detrimental for the US; China supported the protocol since it profited from global greenhouse gas emission reductions without itself being committed to mitigation measures. The goal of the current post-Kyoto climate negotiations is to agree on a longer-term policy that is significantly more ambitious than Kyoto and is regarded as beneficial by all UNFCCC parties (Fig. 1c). However, it is not sufficient that all individual cost-benefit arrows lie in the lower triangle of the cost-benefit plane. The agreement must also be perceived as fair by everyone. This requires that all arrows converge on the same point (Fig. 1d), after appropriate normalization of each countries mitigation and damage costs. This implies, in turn, that a fair balance has been achieved between the different actors' perceptions of equity. 
A clarification of the different perceptions and assessments of a given climate policy by the various UNFCCC parties requires a detailed analysis of the particular situation in each country. Once a general agreement has been reached on the range of global emission pathways to be considered, the modeling task is then to assess the policy portfolios with respect to their effectiveness, efficiency and equitability in realizing the pathways, as seen from the perspective of each country. This is clearly no small task. However, it should be undertaken. A quantitative, objective analysis of the detailed distribution of the global impacts and burdens, carried out by an independent UN panel, would clearly carry significantly greater persuasive weight than the analyses of individual negotiators representing only a single country's interests.

\section{Agreeing on a common climate policy}

Agreeing on a common climate policy is a complex iterative process, in which assessments based on model simulations are only one input. Some rather elementary conclusions can be drawn already without application of sophisticated models. Figure 2 shows the projected mean (BAU) curve (thick black) of the world-averaged $\mathrm{CO}_{2}$ emissions per capita, as well as the level of reductions required within this century if dangerous climate change is to be avoided (defined by the EU and other bodies as a global warming no greater than $2^{\circ} \mathrm{C}$ above the pre-industrial level). Also shown are the estimated BAU per capita emission curves for a few representative countries or regions, together with possible reduction curves that could result from an international climate agreement. It is immediately clear from a first glance that, whatever the details of the agreement, demands that the emerging economies (and still more the developing countries) reduce their BAU emissions significantly in the next decade or two are unfair. As long as the per capita emissions of the affluent industrial economies greatly exceed those of the rest of the world, elementary considerations of equity indicate that the rest of the world will not be willing to adopt significant reduction measures.

Fig. $2 \mathrm{CO}_{2}$ emissions per capita for the world and four representative regions. BAU curves: black; reduction target required to avoid dangerous climate change: red. Possible reduction curves to meet the target: green

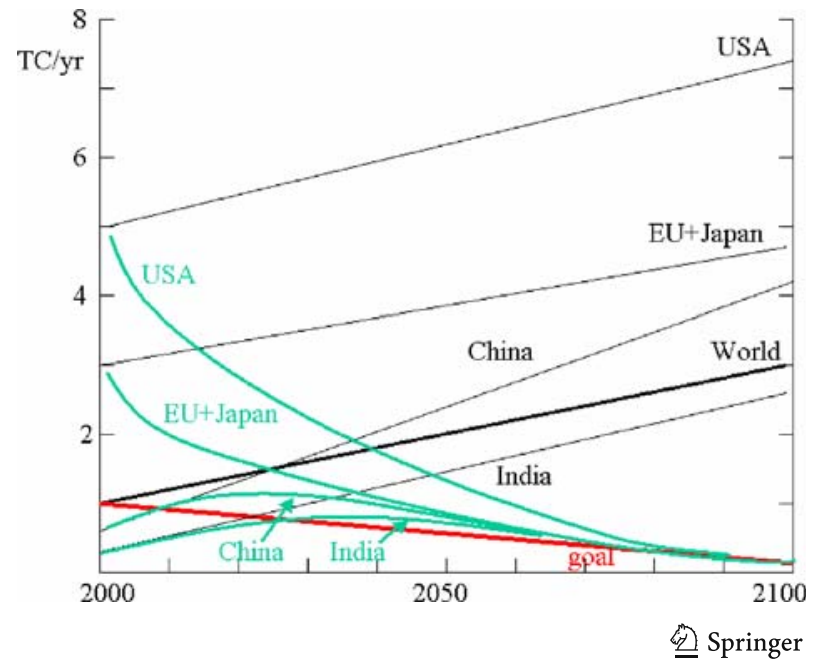


This consideration must be qualified by the lead times required to introduce renewable technologies. However, as long as large disparities persist between the per capita emissions of the industrial countries and the rest of the world, the moral responsibility for resolving this problem lies with the industrial countries. These will need to transfer significant technology know-how and capital resources to the emerging economies to enable them to reduce emissions within the extended, but nonetheless finite time frame indicated in Fig. 2.

A quantification of these elementary considerations by an authoritative international panel would deflate some of the special-interest efforts to ignore the implications of equity in international negotiations, just as the reports of IPCC Working Group 1 led ultimately to the acceptance that climate change is real, and not the "the world's greatest hoax".

A key element in analyses of emission reduction scenarios is the rate of technological change. There is general agreement that the technologies required to restrain global warming below $2^{\circ} \mathrm{C}$ are available already today. Their rate of implementation is a question of cost, emission-reduction capacity and timing. Figure 3 is a sketch of the wedge between the BAU emission curve and the low-carbon emission goal, together with one possible realization of a sequence of renewable technologies that could fill out the wedge (the hump in the 'goal' curve at the beginning of the 21st century is an expression of the time lost in implementing effective climate policies since the establishment of the UNFCCC in 1992).

Technologies that are already market-competitive today, the so-called "no-regrets options" such as increased energy efficiency in production and end-use (combined heat-power generation, improved energy-efficiency of buildings, lighting and transportation, etc.) would enter the market first. This would be followed by technologies that require relatively small carbon prices to penetrate the market (e.g. sinks through reforestation, biomass energy, wind power, hydro-electricity, geothermal energy). Finally, solar technology, either solar thermal or photovoltaic, would need to be introduced on a large scale.

Fig. 3 Time-resolved wedge diagram for the transition from the BAU CO2 emission curve (top boundary) to the low-carbon target (bottom boundary). Higher prices for solar energy present a price barrier at current prices after a few decades. The price barrier can be overcome by efficiency gains through learning by doing and learning by researching

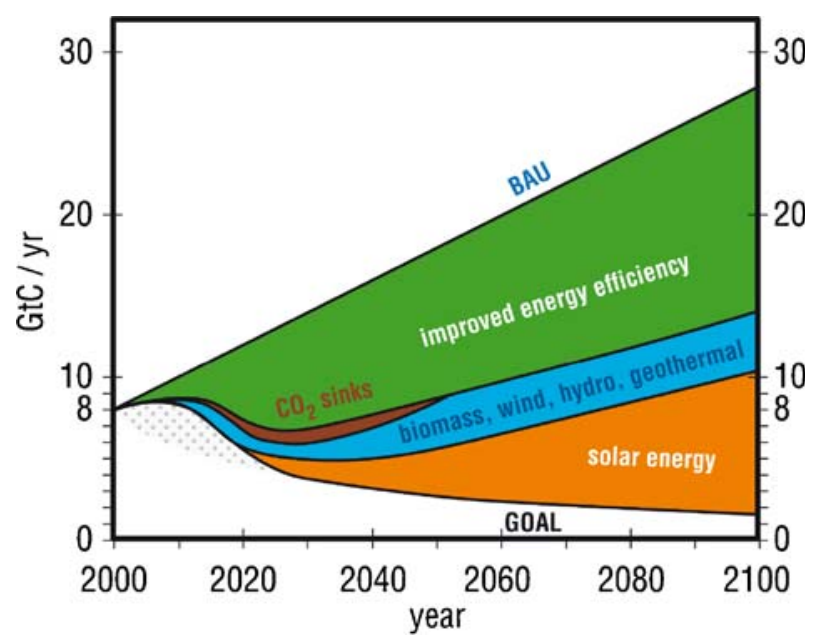


An alternative technology sequence could include extensive carbon capture and sequestration (CCS), which, if proven, would be able to prolong the use of fossil fuel, thereby delaying the need to introduce large scale solar energy technology. Expanding the use of nuclear power is another option. However, the use of a technology with unknown solutions to known risks, instead of renewable energy, offering known solutions to known risks, will remain controversial.

Independent of these alternative technological development paths, a basic limitation of the economically more viable methods of emission reduction is their finite emission-reduction capacity. After a few decades, these technologies alone will be unable to overcome the growth of the BAU emission curve, driven by the rapid growth of the emerging economies. They will need to be supplemented by technologies that sequester $\mathrm{CO}_{2}$, such as biomass energy with $\mathrm{CCS}$, or that involve direct air capture of $\mathrm{CO}_{2}$ and storage. In the longer term, the low-carbon goal can be attained only through the large scale introduction of solar energy, an essentially limitless energy source, if necessary combined with air capture and storage. At current estimates of solar-energy costs, this implies, however, that towards the middle of the century, the costs of emission reduction could rise from present values between $0 \%$ and $2 \%$ of global GDP to values ranging from $2 \%$ to $6 \%$ (Stern 2007).

The cost estimates today are nevertheless expected to drop significantly by the time solar energy enters the market on a large scale through efficiency gains resulting from investments in $\mathrm{R} \& \mathrm{D}$ (learning by researching) and from practical experience (learning by doing). Adopting the optimistic cost reduction assumptions of Edenhofer et al. (2006), obtained by extrapolating historical data for other major energy-technology transitions (water/coal/oil/gas), the Stern Review estimates that the global costs of switching from fossil to low-carbon energy technology are of the order of only $1 \%$ of world GDP. A meta-analysis extrapolating to the 450 ppmv CO2eq stabilization level (the lower end of the Stern range) indicates that the costs vary over a wide range depending on policies, and that over the next 50-100 years, with the potential for improvements through international cooperation, the range includes negative costs (i.e. benefits in addition to the benefits of reducing climate change; Barker and Jenkins 2007). The possibilities of negative costs have been emphasized in both the Stern Review and the IPCC WG3 Report.

To convince governments that the transition to a low-carbon economy can be achieved without major dislocations, the present models of induced technological change will need to be extended to genuine multi-actor models, including the impacts of technological change on employment and other socio-economic factors of immediate concern to policymakers. In this respect, it is encouraging that similar numbers were obtained by Weber et al. (2005) using a multi-actor, dynamic Integrated Assessment model, which simulated the interaction between climate change, endogenous technological change, government-induced technological change, productivity increase, level of employment, savings rates and other economic indices closer to the concerns of policymakers.

The true costs of the long-term transition to a low-carbon economy depend on a number of poorly predictable parameters and have been the subject of considerable debate. It can therefore be helpful to view the larger picture and search for general conclusions that are independent of controversial numbers. Figure 4 shows a sketch of the BAU growth in world GDP, on a log scale, assuming an extremely modest annual growth rate of $1 \%$. As in all BAU projections, the costs of climate change are 
Fig. 4 Sketch of global BAU GDP growth at the (very low rate) of $1 \% /$ year (black curve) and impact on growth of mitigation costs of avoiding dangerous climate change of $1 \%$ (green) and $5 \%$ (red) of global GDP

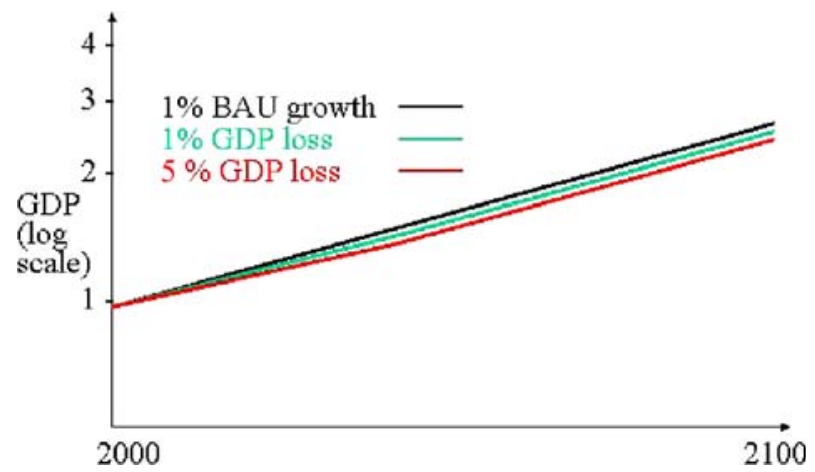

ignored. Also shown in the graph are the impacts of a $1 \%$ and $5 \%$ loss in GDP due to the costs of the transition to a low-carbon economy. The deviation from the BAU curve for the case of a 1\% GDP loss is scarcely discernible on the multi-decadal time scales relevant for climate change. But even for the extremely pessimistic case of a $5 \%$ GDP loss by 2050 (the high-cost outlier relating to the $2^{\circ} \mathrm{C}$ target) quoted in the IPCC Report (Barker and Jenkins 2007) combined with a very sluggish BAU GDP growth rate of $1 \%$, very few would dispute that the insurance premium for avoiding major climate change is a worthwhile investment. This has been stressed also by Azar and Schneider (2002) and Hasselmann et al. (2003).

Thus the central issue is not whether mitigation policies should be undertaken, but which measures should be introduced, at which rate, and how the costs should be distributed.

\section{The ethical problem}

This is not just a technical and economic problem, but equally a moral issue (see, for example, Broome 1992, 2008; Schneider and Lane 2006). Climate change is a much quoted case of Garret Hardin's Tragedy of the Commons (Hardin 1968): all participants have a motivation to avoid implementing remedial actions themselves, free-riding instead on the remedial actions of others. However, climate change is the most extreme example conceivable: it affects everyone on the planet; its consequences are unforeseeable, but could dwarf all other catastrophes of human civilization; there exists only a finite time horizon for action; and there exists no higher authority that can enforce compliance, even if a common policy is agreed upon.

Garret Hardin argued that relying on ethics without enforcement was doomed to failure. His entertaining description of the emotional plight of the good moral citizen duped by unrestrained free-riders undoubtedly appeals to many skeptics of international climate policy. Unfortunately, in the case of climate change, we have no other choice than to rely on human insight and moral responsibility. The creation of an internationally accepted Climate Policy Panel within the UNFCCC, equipped with the necessary modelling and analysis tools, could be an important factor in generating the necessary insights.

Open Access This article is distributed under the terms of the Creative Commons Attribution Noncommercial License which permits any noncommercial use, distribution, and reproduction in any medium, provided the original author(s) and source are credited. 


\section{References}

Ackerman F, Heinzerling L (2004) Priceless: on knowing the price of everything and the value of nothing. The New Press, New York

Azar C, Schneider HS (2002) Are the economic costs of stabilising the atmosphere prohibitive? Ecol Economics 42:73

Barker T (1998) The use of energy-environment-economy models to inform greenhouse gas mitigation policy. Impact Assess Proj Apprais 16(2):133-131

Barker T, Jenkins K (2007) The costs of avoiding dangerous climate change: estimates derived from a meta-analysis of the literature. A Briefing Paper for the United Nations Human Development Report 2007. United Nations Human Development, New York

Beinhocker E (2006) The origin of wealth: evolution, complexity and the radical remaking of economics. Random House Business Books

Broome J (1992) Counting the cost of global warming. White House, Cambridge

Broome J (2008) The ethics of climate change: pay now or pay more later?, Scientific American, http://www.sciam.com/article.cfm?id=the-ethics-of-climate-change\&sc=PR_20080530 May 19, 2008

DeCanio SJ (2003) Economic models of climate change: a critique. MacMillan, New York, p 203

Edenhofer O, Lessmann K, Kemfert C, Grubb M, Köhler J (2006) Induced technological change: exploring its implications for the economics of atmospheric stabilization: synthesis report from the innovative modeling comparison project. The Energy J 27:57-107, Special Issue

Gore $\mathrm{Al}$ (2006) An inconvenient truth. Documentary film

Hardin G (1968) The tragedy of the commons. Science 162:1243-1248

Hasselmann K, Latif M, Hooss G, Azar C, Edenhofer O, Jaeger CC, Johannessen OM, Kemfert C, Welp M, Wokaun A (2003) The challenge of long-term climate change. Science 302:1923-1925

IPCC (2001a) Intergovernmental panel on climate change. In: Houghton JT et al. (eds) Working group 1: the scientific basis. Cambridge University Press, Cambridge

IPCC (2001b) Intergovernmental panel on climate change. In: McCarthy JJ, et al. (eds) Working group 2: impacts, adaptation, and vulnerability. Cambridge University Press, Cambridge

IPCC (2001c) Intergovernmental panel on climate change. In: Metz B, et al. (eds) Working group 3: mitigation. Cambridge University Press, Cambridge

IPCC (2007a) Intergovernmental panel on climate change. Working group 1. Cambridge University Press, Cambridge

IPCC (2007b) Intergovernmental panel on climate change. Working group 2. Cambridge University Press, Cambridge

IPCC (2007c) Intergovernmental panel on climate change. Working group 3. Cambridge University Press, Cambridge

Löschel A (2002) Technological change in economic models of environmental policy: a survey. Ecol Econ 43:105-126

Nordhaus WD (1994) Managing the global commons: the economics of climate change. The MIT Press, Cambridge

Schneider SH, Lane J (2006) Dangers and thresholds in climate change and the implications of justice. In: Adger N (ed) Fairness in adaptation to climate change. MIT, Cambridge

Stern N (2007) The economics of climate change. The Stern Review. Cambridge University Press, Cambridge

van den Bergh JCJM (2004) Optimal climate policy is a utopia: from quantitative to qualitative costbenefit analysis. Ecol Econ 48:385-393

Weber M, Barth V, Hasselmann K (2005) A multi-actor dynamic integrated assessment model (MADIAM) of induced technological change and sustainable economic growth. Ecol Econ 54:306-327

Weitzman ML (2008) On modeling and interpreting the economics of catastrophic climate change. http://www.economics.harvard.edu/faculty/weitzman/files/modeling.pdf

Weyant JP (2000) An introduction to the economics of climate change. PEW centre on global climate change. Batelle, Washington DC 